\title{
Políticas educativas e incorporación de las TIC en la educación superior mexicana
}

\author{
Marisol Muñoz Martínez
}

\begin{abstract}
Resumen
El presente artículo es un análisis sobre la introducción de las Tecnologías de Información y Comunicación (тіс) en el sistema educativo mexicano, a través de la documentación de las políticas educativas. En seguida, se comenta la importancia de las tic como mediadoras en el proceso de aprendizaje, particularmente en la educación superior. Las conclusiones apuntan a centrar la atención en los procesos de aprendizaje más que en la inversión de equipamiento.
\end{abstract}

Palabras clave: educación superior, tecnologías de información y comunicación, políticas públicas educativas, TIC.

\section{Educational Policies and ict Incorporation in Mexican higher education}

\begin{abstract}
This article is an analysis of the introduction of Information and Communication Technologies (IcT) in the Mexican educational system, through the educational policies documentation. The importance of Ict as mediators in the learning process is discussed below, particularly in higher education. The conclusions aim to focus attention on learning processes rather than investment in equipment.
\end{abstract}

Keywords: higher education, information and communication technologies, public education policies, ICT. 
Licenciada en Administración Educativa por la Universidad Pedagógica Nacional (UPN) Unidad Ajusco, recién egresada de la Maestría en Desarrollo Educativo (MDE), en la Línea de Tecnologías de la Información y la Comunicación en Educación.

\section{Introducción}

La actualización y renovación permanente del saber científico y tecnológico, y el sistema económico centrado en la producción, han generado cambios en la forma de elaborar política en México. En particular, han orientado la educación al desarrollo de competencias para dar respuesta a nuevas demandas del mercado laboral, en el contexto de la globalización y la transformación digital de la industria y las organizaciones.

En este marco, el presente artículo centra su atención en la introducción de las Tecnologías de Información y Comunicación (Tic) en el sistema educativo mexicano, a través de la documentación de las políticas educativas. Además, se aborda la importancia de las tecnologías en la educación superior, como mediadoras en el proceso de enseñanza-aprendizaje. Así, surge la cuestión: ¿cuál tendría que ser la prioridad de las políticas educativas en materia de Tıc?

\section{Políticas educativas para la introducción de las TIC en México}

La incorporación y uso de las Tic en las actividades cotidianas humanas ha propiciado que los organismos internacionales se ocupen de la detección de la necesidad de su incorporación al ámbito educativo. Entre dichos organismos se encuentran la Organización de las Naciones Unidas para la Educación, la Ciencia y la Cultura (unesco), la Organización para la Cooperación y el Desarrollo Económico (OCDE) y el Banco Mundial (BM).

Resalta el trabajo realizado por la unEsco para la adopción de las tecnologías en el ámbito educativo. En la década de los noventa constituyó la Comisión Internacional sobre la Educación para el Siglo xxı (1996), presidida por Jaques Delors, y en cuyo informe se indica que, a partir del contexto de un mundo globalizado, multiculturaly con problemas de inequidad, se requiere instrumentar una nueva propuesta de construcción de la educación y el aprendizaje. Además de contar con profesores adecuadamente formados, se considera la incorporación de otros elementos para impartir enseñanza de calidad, dentro de los cuales, el uso de medios de comunicación y de las tecnologías cobran gran relevancia. 
"Políticas educativas e incorporación de las TIC en la educación superior mexicana"

Marisol Muñoz Martínez

Vol. 21, Núm. 6, noviembre-diciembre 2020

Revista Digital Universitaria

En el caso de México, los primeros intentos de introducir los recursos tecnológicos en el sistema educativo mexicano datan del sexenio de Miguel de la Madrid (1982-1988). Con el programa de Telesecundarias, inaugurado en 1986, la Secretaría de Educación Pública (SEP), en el ya mencionado período de gobierno, incorporó la televisión al ámbito educativo, con el fin de poner al alcance los estudios de secundaria para jóvenes residentes en comunidades alejadas de las zonas urbanas.

Posteriormente, entre 1993y 1994, se implementó el Proyecto Introducción de la Computación Electrónica en la Educación Básica (OEEBA-SEP) para educación primaria. Siguieron, de manera consecutiva, el Sistema de Educación Satelital (EDUSAT), inaugurado en 1995, y la Red Nacional de Videoconferencias para la educación, en 1997, con la participación de la Universidad Nacional Autónoma de México (UnAm), el Instituto Politécnico Nacional (IPN) y la Universidad Autónoma Metropolitana (UAM).

Cabe señalar que, en 2003, se hace una adición al artículo seis de la Constitución Política de los Estados Unidos Mexicanos, donde se establece la libertad de expresión y el derecho al acceso de la información, que a letra dice: "el Estado garantizará el acceso a las Tic, así como servicios de radiodifusión y telecomunicaciones, incluido el de banda ancha, e Internet [...]" (Cámara de Diputados del H. Congreso de la Unión, 2008, p.10).

Para inicios del siglo xxI, durante el gobierno de Vicente Fox Quezada (20002006), se impulsó como proyecto sexenal educativo el Programa Enciclomedia (2003), para quinto y sexto grado de primaria. En ese momento se estableció la política de fomento del uso educativo de las tıc en la educación básica, cuya tarea sería "impulsar el uso, expansión, y desarrollo de las Tıc, así como la producción de materiales audiovisuales e informáticos que [...] favorecieran el aprendizaje" (SEP, 2006, pp.13-14). Lo anterior, a través de la distribución computadoras de escritorio, pizarrones blancos, proyectores y un contenido didáctico, que poco se usó por falta de capacitación hacia los profesores, o bien la carencia de servicios básicos como energía eléctrica.

En 2010, durante el gobierno de Felipe Calderón (2006-2012), se implementó el Programa Habilidades Digitales para Todos (HDT), en secundaria, para apoyar el aprendizaje de los estudiantes y ampliar sus competencias para la vida. En el gobierno de Enrique Peña (2012-2018), se propuso ampliar la oferta educativa y mejorar la gestión educativa a través del programa Estrategia Digital Nacional (2014).

Los resultados de los proyectos y programas mencionados dejaron en evidencia las deficiencias de una política educativa, que al parecer no está planeada con objetivos pedagógicos ni estrategias apropiadas para lograrlos. Se hizo una inversión en tecnologías (Severin, 2010), con el supuesto de que, por su sola presencia dentro del aula de clases, se estaría a la vanguardia educativa y, por ende, aportarían al proceso de enseñanza-aprendizaje. 
Como se aprecia, los proyectos y programas implementados en la mayoría de los casos son una combinación de políticas de equipamiento informático y dotación de contenidos digitales para las tareas de enseñanza. Sin embargo, sólo en la minoría se aborda la sustentabilidad de presupuesto dirigido a la capacitación de las y los profesores, y el mantenimiento y actualización del equipamiento. Por ello, es necesario cuestionar el discurso políticamente correcto, para iniciar un abordaje más amplio en la introducción de las Tıc en el ámbito educativo, e involucrar a las y los profesores, administradores educativos, estudiantes universitarios y el currículo.

De acuerdo con lo expuesto, Benavides y Pedró (2007) proponen que las políticas educativas deben definir con antelación un perfil del profesor, y proceder a asegurar la disponibilidad de contenidos y aplicaciones, lo que favorecería la implementación de apoyos dirigidos al área de investigación científica y tecnológica.

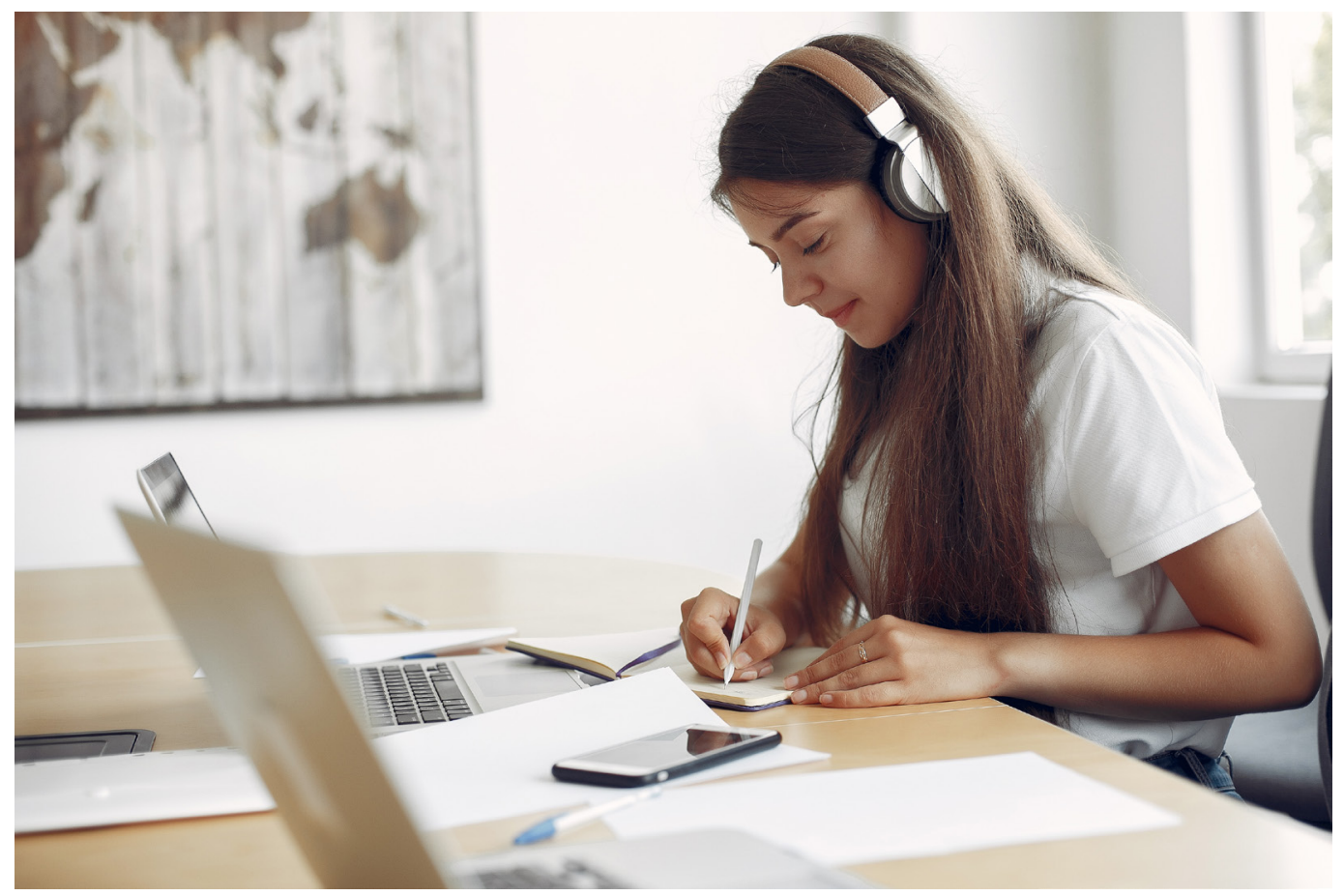

\section{La importancia de las TIC en la educación superior}

LaS Tic han conformado un fenómeno social de gran alcance, que ha transformado las actividades cotidianas de la humanidad. Éstas, asimismo, avanzan de una manera acelerada y sin pauta alguna, por lo que se requiere que los estudiantes universitarios desarrollen ciertas habilidades, destrezas, aptitudes y un juicio propio, que les permitan hacer uso de las tecnologías, en la adquisición, reforzamiento o divulgación del conocimiento.

En la Conferencia Mundial sobre la Educación Superior, se exterioriza que los estudiantes deben asumir la responsabilidad de ser un participante activo 
en el apoderamiento del conocimiento, valores y habilidades necesarios para aprender a: conocer, hacer, trabajar en equipo, a ser solidario, tomar decisiones, resolver problemas, etcétera (UNESCO, 1998). En este sentido, las Instituciones de Educación Superior (IES), en conjunto con las y los profesores juegan un papel relevante. Para Tedesco (2000), la sociedad del conocimiento, en particular la universidad, debe tener en cuenta tres nuevos factores en su acceso al conocimiento:

1. "es necesario educarse toda la vida";

2. la democratización del acceso a los niveles más complejos del conocimiento no puede quedar confiada solamente a la universidad, debido a que la gran mayoría quedaría fuera del proceso de desarrollo personal y social (generándose un nuevo despotismo ilustrado);

3. se deberán encarar los desafíos que plantean las tecnologías de la información a las instituciones y métodos de enseñanza (pp. 77-78).

El objetivo básico de la educación superior, según Tedesco (2000), deberá centrarse en "lograr que las personas aprendan a aprender" (p. 77). Los estudiantes universitarios deberán dominar las operaciones cognitivas fundamentales asociadas a cada dominio del saber y desarrollar actitudes básicas vinculadas con el aprendizaje permanente: curiosidad, interés, espíritu crítico, creatividad, etcétera.

Ante este escenario, es fundamental que las IEs ofrezcan la infraestructura tecnológica, así como capacitación a las y los profesores, con la finalidad de que el estudiante universitario, desarrolle y domine "las destrezas informáticas manejo de software y hardware- e informacionales -gestión del conocimiento, discriminar fuentes formales de consulta, entre otras-" (Edel, 2015, p. 3), estas últimas conocidas también como habilidades, competencias, saberes digitales o capital tecnológico incorporado.

\section{Conclusión}

Las políticas educativas en México muestran una serie de contradicciones. En este artículo se expone una de ellas: el reconocimiento, ante la legislación, del acceso a la información como un derecho humano, pero sin la implementación de políticas de equipamiento informático que el Estado no ha logrado suministrar en todo el sistema educativo mexicano, desde nivel básico a superior.

Incorporar las TIc en la educación debe significar mucho más que una herramienta para mejorar la educación superior en México. Se trata de usarlas con un propósito específico, para generar o reafirmar, en los estudiantes universitarios, conocimientos, habilidades, aptitudes y destrezas, que se necesitan en la sociedad actual. Para ello, se requiere de una política educativa que vaya acompañada de un plan estratégico, que guíe a los líderes educativos institucionales en el proceso de integración de las tecnologías con fines 
educativos. Este proceso debe de ser integral, es decir, debe considerar a los actores educativos: directores, profesores, estudiantes y, dependiendo del nivel educativo, a los padres de familia.

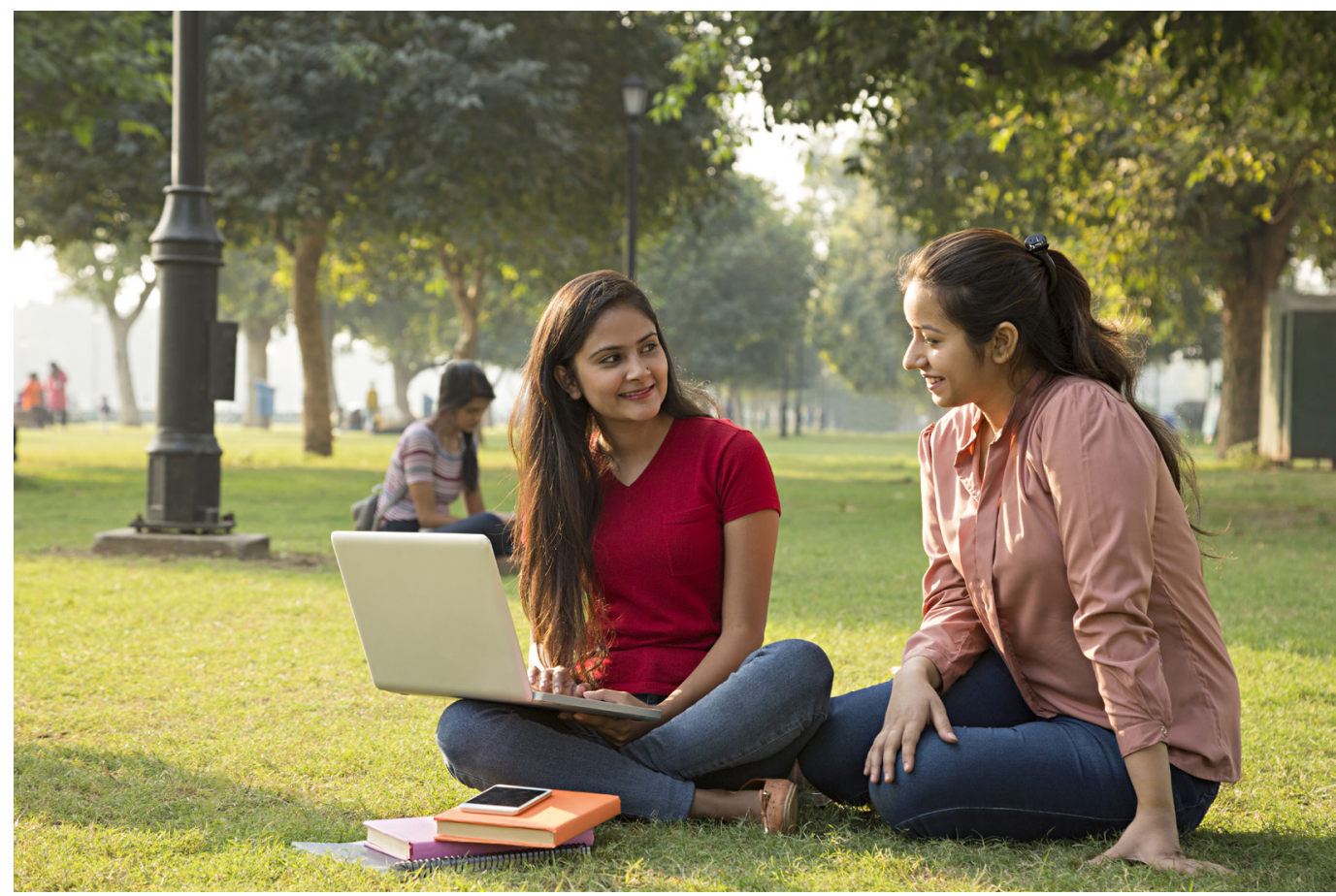

El análisis presentado apunta a centrar la atención en los procesos de aprendizaje sin detrimento a la necesaria inversión en equipamientos. Es necesario reconocer la importancia de la capacitación de las y los profesores en el uso eficaz de las tecnologías como parte de su práctica diaria, en sus procesos de enseñanza-aprendizaje.

Para concluir, las tıc son una realidad, y será determinante ser competente en su uso ante la sociedad de la información y la del conocimiento. Por ello, resulta prioritario su aprendizaje, particularmente en la educación superior, con carácter transversal a cualquier profesión, para estar en consonancia con las demandas laborales.

\section{Referencias}

* Benavides, F. y Pedró, F. (2007). Políticas educativas sobre nuevas tecnologías en los países Iberoamericanos. Revista Iberoamericana de Educación, (45),19-69. http://rieoei.org/rie45a01.htm

* Feixa, C., Fernández-Planells, A., y Figueras-Maz, M. (2016). Generación Hashtag. Los movimientos juveniles en la era de la web social. Revista Latinoamericana de Ciencias Sociales, Niñez y Juventud, 14(1), 107-120. www.scielo.org.co/pdf/rlcs/ v14n1/v14n1a07.pdf 
* Gobierno de México. (27 de agosto de 2008). Constitución Política de los Estados Unidos Mexicanos. Diario oficial de la Federación. http://www.diputados.gob.mx/ LeyesBiblio/pdf/1_270818.pdf

* Organización de las Naciones Unidas para la Educación, la Ciencia y la Cultura (UnEsCo). (1998). Informe mundial sobre la educación. Los docentes y la enseñanza en un mundo en mutación. Santillana.

* Secretaría de Educación Pública. (2006). Libro Blanco, Programa "Enciclomedia" 2006-2012. Secretaría de Educación Pública. https://www.sep.gob.mx/work/ models/sep1/Resource/2959/4/images/LB\%20Enciclomedia.pdf

* Severin, C. E. (2010). Tecnología de la Información y la Comunicación (TIC) en Educación. Marco Conceptual e indicadores (Notas Técnicas \#6). Banco Interamericano de Desarrollo (BID), División de Educación. https://publications.iadb.org/publications/ spanish/document/Tecnolog\%C3\%ADas-de-la-informaci\%C3\%B3n-y-lacomunicaci\%C3\%B3n-(TICs)-en-educaci\%C3\%B3n.pdf

* Tedesco, J. C. (2000). Educar en la sociedad del conocimiento. Fondo Cultura Económica.

\section{Cómo CITAR ESTE ARTículo}

* Muñoz Martínez, Marisol. (2020, noviembre-diciembre). Políticas educativas e incorporación delasTICenlaeducaciónsuperiormexicana. RevistaDigitalUniversitaria (RDU), 21(6). Dol: http://doi.org/10.22201/cuaieed.16076079e.2020.21.6.13 